\title{
On Standing Wave Solutions for Discrete Nonlinear Schrödinger Equations
}

\author{
Guowei Sun \\ Department of Applied Mathematics, Yuncheng University, Yuncheng, Shanxi 044000, China \\ Correspondence should be addressed to Guowei Sun; sunkanry@163.com
}

Received 13 June 2013; Revised 14 July 2013; Accepted 14 July 2013

Academic Editor: Juan J. Nieto

Copyright (C) 2013 Guowei Sun. This is an open access article distributed under the Creative Commons Attribution License, which permits unrestricted use, distribution, and reproduction in any medium, provided the original work is properly cited.

The purpose of this paper is to study a class of discrete nonlinear Schrödinger equations. Under a weak superlinearity condition at infinity instead of the classical Ambrosetti-Rabinowitz condition, the existence of standing waves of the equations is obtained by using the Nehari manifold approach.

\section{Introduction}

The discrete nonlinear Schrödinger (DNLS) equation was first derived in the context of nonlinear optics by Christodoulides and Joseph [1]; see also [2-5]. DNLS equation is one of the most important inherently discrete models, having a crucial role in the modeling of a great variety of phenomena, ranging from solid state and condensed matter physics to biology [6-10]. For example, Davydov [6] studied the equation in molecular biology and Su et al. [10] considered the equation in condensed matter physics. Eilbeck et al. [11] firstly pointed out the universal nature of the discrete nonlinear Schrödinger equation and reported a number of applications.

For the analytical study, many authors studied the existence results of standing wave solutions for DNLS equations. Much of the works concerns the periodic DNLS equations [12-14]. Recently, some authors considered the DNLS equations with infinitely growing potential. Zhang and Pankov $[15,16]$ devoted their efforts to the case of infinitely growing potential and power-like nonlinearity. In all these results, the nonlinearity is supposed to be either positive (self-focusing), or negative (defocusing). Pankov [17] studied the DNLS equatifvons with infinitely growing potential and sign-changing nonlinearity (a mixture of self-focusing and defocusing ones). Pankov and Zhang were concerned with the DNLS equations with infinitely growing potential and saturable nonlinearity in [18].
In this paper, we consider higher-dimensional generalizations of DNLS equation

$$
\begin{array}{r}
i \dot{\phi}_{n}+(\mathscr{A} \phi)_{n}-v_{n} \phi_{n}+\sigma f\left(n, \phi_{n}\right)=0, \\
n=\left(n_{1}, n_{2}, \ldots, n_{m}\right) \in \mathbb{Z}^{m},
\end{array}
$$

where

$$
\begin{aligned}
(\mathscr{A} \phi)_{n}= & \phi_{\left(n_{1}+1, n_{2}, \ldots, n_{m}\right)}+\phi_{\left(n_{1}, n_{2}+1, \ldots, n_{m}\right)} \\
& +\cdots+\phi_{\left(n_{1}, n_{2}, \ldots, n_{m}+1\right)}-2 m \phi_{\left(n_{1}, n_{2}, \ldots, n_{m}\right)} \\
& +\phi_{\left(n_{1}-1, n_{2}, \ldots, n_{m}\right)}+\phi_{\left(n_{1}, n_{2}-1, \ldots ., n_{m}\right)} \\
& +\cdots+\phi_{\left(n_{1}, n_{2}, \ldots, n_{m}-1\right)}
\end{aligned}
$$

and $\sigma= \pm 1$. The parameter $\sigma$ characterizes the focusing properties of the following equation: if $\sigma=1$, the equation is self-focusing, while $\sigma=-1$ corresponds to the defocusing equation.

We assume that the nonlinearity $f(n, u)$ is gauge invariant, that is,

$$
f\left(n, e^{i \theta} u\right)=e^{i \theta} f(n, u), \quad \theta \in \mathbb{R} .
$$

Then we can consider the special solutions of the form $\phi_{n}=e^{-i \omega t} u_{n}$, for any $\omega \in \mathbb{R}$. These solutions are called breather solutions or standing waves, due to their periodic 
time behavior. Inserting the ansatz of a breather solution into (1), it follows that $\phi_{n}$ satisfies the nonlinear system of algebraic equations

$$
-(\mathscr{A} u)_{n}+v_{n} u_{n}-\omega u_{n}-\sigma f\left(n, u_{n}\right)=0, \quad n \in \mathbb{Z}^{m} .
$$

We need the following assumptions.

$\left(V_{1}\right)$ The discrete potential $V=\left\{v_{n}\right\}_{n \in \mathbb{Z}^{m}}$ satisfies

$$
\lim _{|n| \rightarrow \infty} v_{n}=\infty
$$

where $|n|=\left|n_{1}\right|+\left|n_{2}\right|+\cdots+\left|n_{m}\right|$ is the length of multi-index $n$.

$\left(f_{1}\right) \quad f \in C\left(\mathbb{Z}^{m} \times \mathbb{R}, \mathbb{R}\right)$, and there exists $a>0, p \in(2, \infty)$ such that

$$
|f(n, u)| \leq a\left(1+|u|^{p-1}\right), \quad \forall n \in \mathbb{Z}^{m}, u \in \mathbb{R} .
$$

$\left(f_{2}\right) \lim _{|u| \rightarrow 0} f(n, u) / u=0$ uniformly for $n \in \mathbb{Z}^{m}$.

$\left(f_{3}\right) \lim _{|u| \rightarrow \infty} F(n, u) / u^{2}=+\infty$ uniformly for $n \in \mathbb{Z}^{m}$, where $F(n, u)$ is the primitive function of $f(n, u)$, that is,

$$
F(n, u)=\int_{0}^{u} f(n, s) d s .
$$

$\left(f_{4}\right) u \mapsto f(n, u) /|u|$ is strictly increasing on $(-\infty, 0)$ and $(0, \infty)$.

We are concerned with the existence of ground state solutions, that is, solutions corresponding to the least positive critical value of the variational functional. To obtain the existence of ground states, usually besides the growth condition on the nonlinearity and a Nehari type condition, the following classical Ambrosetti-Rabinowitz superlinear condition (see, e.g., [19]) is assumed:

$$
0<\mu F(n, u) \leq f(n, u) u, \quad \text { for some } \mu>2, u \neq 0
$$

It is easy to see that (8) implies that $F(n, u) \geq C|u|^{\mu}$, for some constant $C>0$ and $|u| \geq 1$.

In this paper, instead of (8) we assume the superquadratic condition $\left(f_{3}\right)$. It is easy to see that $(8)$ implies $\left(f_{3}\right)$. It is well known that many nonlinearities such as

$$
f(n, u)=u \ln (1+|u|)
$$

do not satisfy (8). A crucial role that (8) plays is to ensure the boundedness of Palais-Smale sequences.

This paper is organized as follows. In Section 2, we establish the variational framework associated with (4). We then present the main results of this paper and compare them with the existing ones. Section 3 is devoted to prove some useful lemmas, and the proof of the main results is completed in Section 4.

\section{Preliminaries}

In order to apply the critical point theory, we will establish the corresponding variational framework associated with (4).

For some positive integer $m$, we consider the real sequence spaces

$$
\begin{array}{r}
l^{p} \equiv l^{p}\left(\mathbb{Z}^{m}\right)=\left\{u=\left\{u_{n}\right\}_{n \in \mathbb{Z}^{m}}: \forall n \in \mathbb{Z}^{m}, u_{u} \in \mathbb{R},\right. \\
\left.\|u\|_{l^{p}}=\left(\sum_{n \in \mathbb{Z}^{m}}\left|u_{n}\right|^{p}\right)^{1 / p}<\infty\right\} .
\end{array}
$$

Then the following embedding between $l^{p}$ spaces holds:

$$
l^{q} \subset l^{p}, \quad\|u\|_{l^{p}} \leq\|u\|_{l^{q}}, \quad 1 \leq q \leq p \leq \infty .
$$

Let

$$
L=-\mathscr{A}+V
$$

which is a self-adjoint operator defined on $l^{p}\left(\mathbb{Z}^{m}\right)($ see $[20])$.

Define the space

$$
E:=\left\{u \in l^{2}\left(\mathbb{Z}^{m}\right): L^{1 / 2} u \in l^{2}\left(\mathbb{Z}^{m}\right)\right\} .
$$

Then $E$ is a Hilbert space equipped with the norm

$$
\|u\|=\left\|L^{1 / 2} u\right\|_{l^{2}\left(\mathbb{Z}^{m}\right)} .
$$

Now we consider the variational functional $J$ defined on E by

$$
J(u)=\frac{1}{2}((L-\omega) u, u)-\sigma \sum_{n \in \mathbb{Z}^{m}} F\left(n, u_{n}\right),
$$

where $(\cdot, \cdot)$ is the inner product in $l^{2}$. Then $J \in C^{1}(E, \mathbb{R})$. And for the derivative of $J$, we have the following formula:

$$
\left(J^{\prime}(u), v\right)=((L-\omega) u, v)-\sigma \sum_{n \in \mathbb{Z}^{m}} f\left(n, u_{n}\right) v_{n}, \quad \forall v \in E .
$$

Equation (16) implies that (4) is the corresponding EulerLagrange equation for $J$. Thus, we have reduced the problem of finding a nontrivial solution of (4) to that of seeking a nonzero critical point of the functional $J$ on $E$.

The following lemma plays an important role in this paper; it was established in [20].

Lemma 1. If $V$ satisfies the condition $\left(V_{1}\right)$, then

(1) for any $2 \leq p \leq \infty$, the embedding map from $E$ into $l^{p}\left(\mathbb{Z}^{m}\right)$ is compact,

(2) the spectrum $\sigma(L)$ is discrete and consists of simple eigenvalues accumulating to $+\infty$.

By Lemma 1, we may assume that $\lambda_{1}$ is the smallest eigenvalue of $L$, that is

$$
\lambda_{1}=\inf \sigma(L) .
$$

Now we are ready to state the main results. 
Theorem 2. Suppose that conditions $\left(V_{1}\right)$ and $\left(f_{1}\right)-\left(f_{4}\right)$ are satisfied. Then one has the following conclusions.

(1) If $\sigma=-1, \omega \leq \lambda_{1}$, (4) has no nontrivial solution.

(2) If $\sigma=1, \omega<\lambda_{1}$, (4) has a nontrivial ground state solution.

(3) If $\sigma=1, \omega<\lambda_{1}$, and $f(n, u)$ is odd in $u$ for each $n \in \mathbb{Z}^{m}$, (4) has infinitely many pairs of solutions $\pm u^{(k)}$ in $E$.

Remark 3. In [20], the author considered the following DNLS equation:

$$
L u_{n}-\omega u_{n}-\sigma \gamma_{n} f\left(u_{n}\right)=0
$$

where there exists a positive constant $\bar{\gamma}$, such that for any $n \in \mathbb{Z}^{m}, 0<\gamma_{n} \leq \bar{\gamma}$. Clearly, (18) corresponds (4) if we let $f(n, u)=\gamma_{n} f(u)$. Therefore, (18) is a special case of (4).

In [20], the nonlinearity $f \in C^{1}(\mathbb{R})$ satisfies the following condition:

$$
0<(q-1) f(u) u \leq f^{\prime}(u) u^{2}, \quad \forall u \neq 0,2<q<\infty,
$$

which implies (8). So it is a stronger condition than $\left(f_{3}\right)$. Therefore, our results generalize the corresponding ones.

Remark 4. In [16], the authors also considered (18) and assumed that the nonlinearity $f \in C^{1}(\mathbb{R})$ satisfies the classical Ambrosetti-Rabinowitz superlinear condition (8). Clearly, it is a stronger condition than $\left(f_{3}\right)$.

Since $\omega<\lambda_{1}$, we may introduce an equivalent norm in $E$ by setting

$$
\|u\|^{2}:=((L-\omega) u, u)
$$

and then the functional $J$ can be rewritten as

$$
J(u)=\frac{1}{2}\|u\|^{2}-\sigma \sum_{n \in \mathbb{Z}^{m}} F\left(n, u_{n}\right)
$$

To prove the multiplicity results, we need the following lemma.

Lemma 5 (see [21]). Let $S=\{w \in E:\|w\|=1\}$. If $E$ is a infinite-dimensional Hilbert space, $\Phi \in C^{1}(S, \mathbb{R})$ is even and bounded below and satisfies the Palais-Smale condition. Then $\Phi$ has infinitely many pairs of critical points.

\section{Some Lemmas}

In this section, we always assume that $\sigma=1$.

We define the Nehari manifold

$$
\mathcal{N}=\left\{u \in E \backslash\{0\}: J^{\prime}(u) u=0\right\} .
$$

To prove the main results, we need some lemmas.
Lemma 6. Suppose that conditions $\left(V_{1}\right)$ and $\left(f_{1}\right)-\left(f_{4}\right)$ are satisfied. Then one has

(1) $F(n, u)>0$ and $(1 / 2) f(n, u) u>F(n, u)$ for all $u \neq 0$,

(2) $J(u)>0$, for all $u \in \mathcal{N}$.

Proof. (1) From $\left(f_{2}\right)$ and $\left(f_{4}\right)$, it is easy to get that

$$
F(n, u)>0, \quad \forall u \neq 0 .
$$

By $\left(f_{4}\right)$, we have

$$
\frac{u}{2} f(n, u)-\int_{0}^{u} f(n, s) d s>\frac{u}{2} f(n, u)-\frac{f(n, u)}{u} \int_{0}^{u} s d s=0 .
$$

So $(1 / 2) f(n, u) u>F(n, u)$ for all $u \neq 0$.

(2) For all $u \in \mathcal{N}$, by (1), we have

$$
\begin{aligned}
J(u) & =J(u)-\frac{1}{2} J^{\prime}(u) u \\
& =\sigma \sum_{n \in \mathbb{Z}^{m}}\left(\frac{1}{2} f(n, u) u-F(n, u)\right)>0 .
\end{aligned}
$$

Lemma 7. Suppose that conditions $\left(V_{1}\right)$ and $\left(f_{1}\right)-\left(f_{4}\right)$ are satisfied, and let $I(u)=\sum_{n \in \mathbb{Z}^{m}} F\left(n, u_{n}\right)$. Then one has the following.

(1) $I^{\prime}(u)=o(\|u\|)$ as $u \rightarrow 0$.

(2) $s \mapsto I^{\prime}(s u) u / s$ is strictly increasing for all $u \neq 0$ and $s>0$.

(3) $I(s u) / s^{2} \rightarrow \infty$ uniformly for $u$ on the weakly compact subsets of $E \backslash\{0\}$, as $s \rightarrow \infty$.

Proof. (1) and (2) are easy to be shown from $\left(f_{2}\right)$ and $\left(f_{4}\right)$, respectively. Next, we verify (3). Let $W \subset E \backslash\{0\}$ be weakly compact and let $\left\{u^{(k)}\right\} \subset W$. It suffices to show that if $s^{(k)} \rightarrow \infty$ as $k \rightarrow \infty$, then so does a subsequence of $I\left(s^{(k)} u^{(k)}\right) /\left(s^{(k)}\right)^{2}$. Passing to a subsequence if necessary, $u^{(k)} \rightarrow u \in E \backslash\{0\}$ and $u_{n}^{(k)} \rightarrow u_{n}$ for every $n$, as $k \rightarrow \infty$.

Since $\left|s^{(k)} u_{n}^{(k)}\right| \rightarrow \infty$ and $u^{(k)} \neq 0$, by $\left(f_{3}\right)$ and (23), we have

$$
\frac{I\left(s^{(k)} u^{(k)}\right)}{\left(s^{(k)}\right)^{2}}=\sum_{n \in \mathbb{Z}^{m}} \frac{F\left(n, s^{(k)} u_{n}^{k}\right)}{\left(s^{(k)} u_{n}^{(k)}\right)^{2}}\left(u_{n}^{(k)}\right)^{2} \longrightarrow \infty \quad \text { as } k \longrightarrow \infty .
$$

Lemma 8. Under the assumptions $\left(V_{1}\right)$ and $\left(f_{1}\right)-\left(f_{4}\right)$, for each $w \in E \backslash\{0\}$, there exists a unique $s_{w}>0$ such that $s_{w} w \in \mathcal{N}$.

Proof. Let $g(s):=J(s w), s>0$. Note that

$$
g^{\prime}(s)=J^{\prime}(s w) w=s\left(\|w\|^{2}-s^{-1} I^{\prime}(s w) w\right),
$$

and from (2) of Lemma 7, then there exists a unique $s_{w}$, such that $g^{\prime}(s)>0$ whenever $0<s<s_{w}, g^{\prime}(s)<0$ whenever $s>s_{w}$, and $g^{\prime}\left(s_{w}\right)=J^{\prime}\left(s_{w} w\right) w=0$. So $s_{w} w \in \mathcal{N}$. 
Remark 9. By (1) and (3) of Lemma 7, $g(s)>0$ for $s>0$ small and $g(s)<0$ for $s>0$ large. Together with Lemma 8, we have that $s_{w}$ is a unique maximum of $g(s)$ and $s_{w} w$ is the unique point on the ray $s \mapsto s w(s>0)$ which intersects with $\mathcal{N}$. That is, $u \in \mathcal{N}$ is the unique maximum of $J$ on the ray. Therefore, we may define the mapping $\widehat{m}: E \backslash\{0\} \rightarrow \mathcal{N}$ and $m: S \rightarrow \mathcal{N}$ by setting

$$
\widehat{m}(w):=s_{w} w, \quad m:=\left.\widehat{m}\right|_{S}
$$

where $S=\{u \in E:\|u\|=1\}$.

Lemma 10. For each compact subset $\mathscr{V} \subset S$, there exists a constant $C_{\mathscr{V}}$ such that $s_{w} \leq C_{\mathscr{V}}$ for all $w \in \mathscr{V}$.

Proof. Suppose that, by contradiction, $s_{w}^{(k)} \rightarrow \infty$ as $k \rightarrow$ $\infty$. By Lemma 6 and $\left(f_{3}\right)$, we have

$$
\begin{aligned}
0< & \frac{J\left(s_{w}^{(k)} w\right)}{\left(s_{w}^{(k)}\right)^{2}}=\frac{1}{2}\|w\|^{2} \\
& -\sum_{n \in \mathbb{Z}^{m}} \frac{F\left(n, s_{w}^{(k)} w_{n}\right)}{\left(s_{w}^{(k)}\right)^{2} w_{n}^{2}} w_{n}^{2} \longrightarrow-\infty, \quad \text { as } k \longrightarrow \infty
\end{aligned}
$$

This is a contradiction.

Lemma 11. (1) The mapping $\widehat{m}$ is continuous.

(2) The mapping $m$ is a homeomorphism between $S$ and $\mathcal{N}$, and the inverse of $m$ is given by $m^{-1}(u)=u /\|u\|$.

Proof. (1) Suppose that $w_{n} \rightarrow w \neq 0$. Since $\widehat{m}(t u)=\widehat{m}(u)$ for each $t>0$, we may assume that $w_{n} \in S$ for all $n$. Write $\widehat{m}\left(w_{n}\right)=s_{w_{n}} w_{n}$. By Lemmas 8 and $10,\left\{s_{w_{n}}\right\}$ is bounded, and hence $s_{w_{n}} \rightarrow s>0$ after passing to a subsequence if needed. Since $\mathcal{N}$ is closed and $\widehat{m}\left(w_{n}\right)=s_{w_{n}} w_{n} \rightarrow s w$, sw $\epsilon$ $\mathcal{N}$. Hence $s w=s_{w} w=\widehat{m}(w)$ by the uniqueness of $s_{w}$ of Lemma 8. (2) This is an immediate consequence of (1).

Lemma 12. J satisfies the Palais-Smale condition on $\mathcal{N}$.

Proof. Let $\left\{u^{(k)}\right\} \subset \mathcal{N}$ be a sequence such that $J\left(u^{(k)}\right) \leq d$ for some $d>0$ and $J^{\prime}\left(u^{(k)}\right) \rightarrow 0$ as $k \rightarrow \infty$.

Firstly, we prove that $\left\{u^{(k)}\right\}$ is bounded. In fact, if not, we may assume by contradiction that $\left\|u^{(k)}\right\| \rightarrow \infty$ as $k \rightarrow \infty$. Let $v^{(k)}=u^{(k)} /\left\|u^{(k)}\right\|$. Then there exists a subsequence, still denoted by the same notation, such that $v^{(k)} \rightarrow v$ in $E$ as $k \rightarrow$ $\infty$. have

Suppose that $v=0$. For every $s>0$, from Remark 9, we

$$
d \geq J\left(u^{(k)}\right) \geq J\left(s v^{(k)}\right)=\frac{1}{2} s^{2}\left\|v^{(k)}\right\|^{2}-I\left(s v^{(k)}\right) \longrightarrow \frac{1}{2} s^{2}
$$

This is a contradiction if $s \geq \sqrt{2 d}$. Therefore, $v \neq 0$.
According to Lemma 7(3), we have

$$
\begin{aligned}
0 & \leq \frac{J\left(u^{(k)}\right)}{\left\|u^{(k)}\right\|^{2}} \\
& =\frac{1}{2}-\frac{I\left(\left\|u^{(k)}\right\| v^{(k)}\right)}{\left\|u^{(k)}\right\|^{2}} \longrightarrow-\infty, \quad k \longrightarrow \infty,
\end{aligned}
$$

a contradiction again. Thus, $\left\{u^{(k)}\right\}$ is bounded.

Finally, we show that there exists a convergent subsequence of $\left\{u^{(k)}\right\}$. Actually, there exists a subsequence, still denoted by the same notation, such that $u^{(k)} \rightarrow u$. By Lemma 1 , for any $2 \leq q \leq \infty$, then

$$
u^{(k)} \longrightarrow u \text { in } l^{q}\left(\mathbb{Z}^{m}\right)
$$

Note that

$$
\begin{aligned}
\| u^{(k)}- & u\left\|^{2}-\omega\right\| u^{(k)}-u \|_{2}^{2} \\
= & \left(J^{\prime}\left(u^{(k)}\right)-J^{\prime}(u),\left(u^{(k)}-u\right)\right) \\
& +\sum_{n \in \mathbb{Z}^{m}}\left(f\left(n, u_{n}^{(k)}\right)-f\left(n, u_{n}\right)\right)\left(u_{n}^{(k)}-u_{n}\right) .
\end{aligned}
$$

The first term $\left(J^{\prime}\left(u^{(k)}\right)-J^{\prime}(u),\left(u^{(k)}-u\right)\right) \rightarrow 0$ as $k \rightarrow \infty$ because of the weak convergence.

$\operatorname{By}\left(f_{1}\right)$ and $\left(f_{2}\right)$, it is easy to show that for any $\varepsilon>0$, there exists $c_{\varepsilon}>0$, such that

$$
|f(n, u)| \leq \varepsilon|u|+c_{\varepsilon}|u|^{p-1}, \quad|F(n, u)| \leq \varepsilon|u|^{2}+c_{\varepsilon}|u|^{p} .
$$

Then,

$$
\begin{gathered}
\sum_{n \in \mathbb{Z}^{m}}\left(f\left(n, u_{n}^{(k)}\right)-f\left(n, u_{n}\right)\right)\left(u_{n}^{(k)}-u_{n}\right) \\
\leq \sum_{n \in \mathbb{Z}^{m}}\left[\varepsilon\left(\left|u_{n}^{(k)}\right|+\left|u_{n}\right|\right)\right. \\
\left.+c_{\varepsilon}\left(\left|u_{n}^{(k)}\right|^{p-1}+\left|u_{n}\right|^{p-1}\right)\right]\left|u_{n}^{(k)}-u_{n}\right| \\
\leq \varepsilon\left(\left\|u^{(k)}\right\|_{l^{2}}+\|u\|_{l^{2}}\right)\left\|u^{(k)}-u\right\|_{l^{2}} \\
+c_{\varepsilon}\left(\left\|u^{(k)}\right\|_{l^{p}}^{p-1}+\|u\|_{l^{p}}^{p-1}\right)\left\|u^{(k)}-u\right\|_{l^{p}} .
\end{gathered}
$$

Combining (32) and the boundedness of $\left\{u^{(k)}\right\}$, the above inequality implies

$$
\begin{aligned}
& \sum_{n \in \mathbb{Z}^{m}}(\left.f\left(n, u_{n}^{(k)}\right)-f\left(n, u_{n}\right)\right) \\
& \quad \times\left(u_{n}^{(k)}-u_{n}\right) \longrightarrow 0 \text { as } k \longrightarrow \infty .
\end{aligned}
$$

It follows from (33) that $u^{(k)} \rightarrow u$ in $E$; that is, $J$ satisfies Palais-Smale condition.

The proof is complete. 
Now we define the functional $\widehat{\Psi}: E \backslash\{0\} \rightarrow \mathbb{R}$ and $\Psi:$ $S \rightarrow \mathbb{R}$ by

$$
\widehat{\Psi}(w):=J(\widehat{m}(w)), \quad \Psi(w):=\left.\widehat{\Psi}\right|_{S}
$$

Lemma 13. (1) $\widehat{\Psi} \in C^{1}(E \backslash\{0\}, \mathbb{R})$, and

$$
\widehat{\Psi}^{\prime}(w) z=\frac{\|\widehat{m}(w)\|}{\|w\|} J^{\prime}(\widehat{m}(w)) z \quad \forall w, z \in E, w \neq 0 .
$$

(2) $\Psi \in C^{1}(S, \mathbb{R})$, and

$$
\begin{array}{r}
\Psi^{\prime}(w)=\|m(w)\| J^{\prime}(m(w)) z \\
\forall z \in T_{w}(S)=\{v \in E:(w, v)=0\} .
\end{array}
$$

(3) $\left\{w_{n}\right\}$ is a Palais-Smale sequence for $\Psi$ if and only if $\left\{m\left(w_{n}\right)\right\}$ is a Palais-Smale sequence for $J$.

(4) $w$ is a critical point of $\Psi$ if and only if $m(w)$ is a nontrivial critical point of J. Moreover, the corresponding values of $\Psi$ and $J$ coincide and $\inf _{S} \Psi=\inf _{\mathcal{N}} J$.

Proof. (1) Let $w \in E \backslash\{0\}$ and $z \in E$. By Remark 9 and the mean value theorem, we obtain

$$
\begin{aligned}
\widehat{\Psi}(w+t z)-\widehat{\Psi}(w) & =J\left(s_{w+t z}(w+t z)\right)-J\left(s_{w} w\right) \\
& \leq J\left(s_{w+t z}(w+t z)\right)-J\left(s_{w+t z}(w)\right) \\
& =J^{\prime}\left(s_{w+t z}\left(w+\tau_{t} t z\right)\right) s_{w+t z} t z
\end{aligned}
$$

where $|t|$ is small enough and $\tau_{t} \in(0,1)$. Similarly,

$$
\begin{aligned}
\widehat{\Psi}(w+t z)-\widehat{\Psi}(w) & =J\left(s_{w+t z}(w+t z)\right)-J\left(s_{w} w\right) \\
& \geq J\left(s_{w}(w+t z)\right)-J\left(s_{w}(w)\right) \\
& =J^{\prime}\left(s_{w}\left(w+\eta_{t} t z\right)\right) s_{w} t z
\end{aligned}
$$

where $\eta_{t} \in(0,1)$. From the proof of Lemma 11, the function $w \mapsto s_{w}$ is continuous, combining these two inequalities that

$$
\begin{aligned}
\lim _{t \rightarrow 0} \frac{\widehat{\Psi}(w+t z)-\widehat{\Psi}(w)}{t} \\
\quad=s_{w} J^{\prime}\left(s_{w} w\right) z \\
\quad=\frac{\|\widehat{m}(w)\|}{\|w\|} J^{\prime}(\widehat{m}(w)) z .
\end{aligned}
$$

Hence the Gâteaux derivative of $\widehat{\Psi}$ is bounded linear in $z$ and continuous in $w$. It follows that $\widehat{\Psi}$ is a class of $C^{1}$ (see [19, Proposition 1.3]). $\widehat{m}(w)$.
(3) Let $\left\{w_{n}\right\}$ be a Palais-Smale sequence for $\Psi$, and let $u_{n}=$ $m\left(w_{n}\right) \in \mathcal{N}$. Since for every $w_{n} \in S$ we have an orthogonal splitting $E=T_{w_{n}} S \oplus \mathbb{R} w_{n}$, using (2) we have

$$
\begin{aligned}
& \left\|\Psi^{\prime}\left(w_{n}\right)\right\|=\sup _{\substack{z \in T_{w_{n}} S \\
\|z\|=1}} \Psi^{\prime}\left(w_{n}\right) z \\
& =\left\|m\left(w_{n}\right)\right\| \sup _{\substack{z \in T_{w_{n}} S \\
\|z\|=1}} J^{\prime}\left(m\left(w_{n}\right)\right) z \\
& =\left\|u_{n}\right\| \sup _{\substack{z \in T_{w_{n}} S \\
\|z\|=1}} J^{\prime}\left(u_{n}\right) z .
\end{aligned}
$$

Using (2) again, then

$$
\begin{aligned}
\left\|\Psi^{\prime}\left(w_{n}\right)\right\| & \leq\left\|u_{n}\right\|\left\|J^{\prime}\left(u_{n}\right)\right\| \\
& =\left\|u_{n}\right\| \sup _{\substack{z \in T_{w_{n}} S, t \in \mathbb{R} \\
z+t w \neq 0}} \frac{J^{\prime}\left(u_{n}\right)(z+t w)}{\|z+t w\|} \\
& \leq\left\|u_{n}\right\| \sup _{z \in T_{w_{n}} S \backslash\{0\}} \frac{J^{\prime}\left(u_{n}\right)(z)}{\|z\|}=\left\|\Psi^{\prime}\left(w_{n}\right)\right\| .
\end{aligned}
$$

Therefore,

$$
\left\|\Psi^{\prime}\left(w_{n}\right)\right\|=\left\|u_{n}\right\|\left\|J^{\prime}\left(u_{n}\right)\right\| .
$$

According to Lemma 6, for $u_{n} \in \mathcal{N}, J\left(u_{n}\right)>0$, so there exists a constant $c>0$ such that $J\left(u_{n}\right)>c$. And since $c \leq J\left(u_{n}\right)=(1 / 2)\left\|u_{n}\right\|^{2}-I\left(u_{n}\right) \leq(1 / 2)\left\|u_{n}\right\|^{2},\left\|u_{n}\right\| \geq \sqrt{2 c}$. Together with Lemma $12, \sqrt{2 c} \leq\left\|u_{n}\right\| \leq \sup _{n}\left\|u_{n}\right\|<\infty$. Hence $\left\{w_{n}\right\}$ is a Palais-Smale sequence for $\Psi$ if and only if $\left\{u_{n}\right\}$ is a Palais-Smale sequence for $J$.

(4) By (45), $\Psi^{\prime}(w)=0$ if and only if $J^{\prime}(m(w))=0$. The other part is clear.

\section{Proof of Main Results}

Proof of Theorem 2. (1) If $\sigma=-1, \omega \leq \lambda_{1}$, we suppose that (4) has a nontrivial solution $u \in E$. Then $u$ is a nonzero critical point of $J$ in $E$ and $J^{\prime}(u)=0$. But

$$
\begin{aligned}
\left(J^{\prime}(u), u\right) & =((L-\omega) u, u)-\sigma \sum_{n \in \mathbb{Z}^{m}} f\left(n, u_{n}\right) u_{n} \\
& \geq \sum_{n \in \mathbb{Z}^{m}} f\left(n, u_{n}\right) u_{n}>0 .
\end{aligned}
$$

This is a contradiction.

(2) If $\sigma=1, \omega<\lambda_{1}$. We firstly show that $\Psi$ satisfies the Palais-Smale condition.

Let $\left\{w^{(k)}\right\}$ be a Palais-Smale sequence for $\Psi$; then $\left\{u^{(k)}\right\}$ is a Palais-Smale sequence for $J$ by Lemma 13(3), where $u^{(k)}:=$ $m\left(w^{(k)}\right) \in \mathcal{N}$. From Lemma $12, u^{(k)} \rightarrow u$ after passing to a subsequence and $w^{(k)} \rightarrow m^{-1}(u)$, so $\Psi$ satisfies the PalaisSmale condition.

Let $\left\{w^{(k)}\right\} \subset S$ be a minimizing sequence for $\Psi$. By Ekeland's variational principle, we may assume that $\Psi^{\prime}\left(w^{(k)}\right) \rightarrow$ 0 as $k \rightarrow \infty$, so $\left\{w^{(k)}\right\}$ is a Palais-Smale sequence for $\Psi$. 
By Palais-Smale condition, $w^{(k)} \rightarrow w$ after passing to a subsequence if needed. Hence $w$ is a minimizer for $\Psi$ and therefore a critical point of $\Psi$, and then $u=m(w)$ is a critical point of $J$ and is also a minimizer for $J$ by Lemma 13 . Therefore, $u$ is a ground state solution of (4).

(3) If $\sigma=1, \omega<\lambda_{1}$, and $f(n, u)$ is odd in $u$ for each $n \in$ $\mathbb{Z}^{m}$, then $J$ is even and so is $\Psi$. Since $\inf _{S} \Psi=\inf _{\mathcal{N}} J>0$ and $\Psi$ satisfies the Palais-Smale condition, $\Psi$ has infinitely many pairs of critical points by Lemma 5. It follows that (4) has infinitely many pairs of solutions $\pm u^{(k)}$ in $E$ from Lemma 13.

This completes Theorem 2.

\section{Acknowledgments}

This work is supported by Program for the National Natural Science Foundation of China (no. 11071283) and Yuncheng University Science Foundation (nos. JY-2011026, JY-2011038, JY-2011039, and JC-2009024).

\section{References}

[1] D. Christodoulides and R. Joseph, "Discrete self-focusing in nonlinear arrays of coupled waveguides," Optics Letters, vol. 13, pp. 794-796, 1988.

[2] A. B. Aceves, "Optical gap solitons: past, present, and future; theory and experiments," Chaos, vol. 10, no. 3, pp. 584-589, 2000.

[3] J. C. Bronski, M. Segev, and M. I. Weinstein, "Mathematical frontiers in optical solitons," Proceedings of the National Academy of Sciences of the United States of America, vol. 98, no. 23, pp. 12872-12873, 2001.

[4] A. A. Sukhorukov and Y. S. Kivshar, "Generation and stability of discrete gap solitons," Optics Letters, vol. 28, no. 23, pp. 23452347, 2003.

[5] M. I. Weinstein, "Excitation thresholds for nonlinear localized modes on lattices," Nonlinearity, vol. 12, no. 3, pp. 673-691, 1999.

[6] A. S. Davydov, "The theory of contraction of proteins under their excitation," Journal of Theoretical Biology, vol. 38, no. 3, pp. 559-569, 1973.

[7] S. Flach and A. V. Gorbach, "Discrete breathers-advances in theory and applications," Physics Reports, vol. 467, no. 1-3, pp. 1-116, 2008.

[8] S. Flach and C. R. Willis, "Discrete breathers," Physics Reports, vol. 295, no. 5, pp. 181-264, 1998.

[9] D. Hennig and G. P. Tsironis, "Wave transmission in nonlinear lattices," Physics Reports, vol. 307, no. 5-6, pp. 333-432, 1999.

[10] W. Su, J. Schieer, and A. Heeger, "Solions in polyacetylene," Physical Review Letters, vol. 42, pp. 1698-1701, 1979.

[11] J. C. Eilbeck, P. S. Lomdahl, and A. C. Scott, "The discrete selftrapping equation," Physica D. Nonlinear Phenomena, vol. 16, no. 3, pp. 318-338, 1985.

[12] A. Mai and Z. Zhou, "Ground state solutions for the periodic discrete nonlinear Schrödinger equations with superlinear nonlinearities," Abstract and Applied Analysis, vol. 2013, Article ID 317139, 11 pages, 2013.

[13] A. Pankov, "Gap solitons in periodic discrete nonlinear Schrödinger equations," Nonlinearity, vol. 19, no. 1, pp. 27-40, 2006.

[14] A. Pankov, "Gap solitons in periodic discrete nonlinear Schrödinger equations. II. A generalized Nehari manifold approach," Discrete and Continuous Dynamical Systems. Series A, vol. 19, no. 2, pp. 419-430, 2007.

[15] G. Zhang and A. Pankov, "Standing waves of the discrete nonlinear Schrödinger equations with growing potentials," Communications in Mathematical Analysis, vol. 5, no. 2, pp. 38 49, 2008.

[16] G. Zhang and A. Pankov, "Standing wave solutions of the discrete non-linear Schrödinger equations with unbounded potentials. II," Applicable Analysis, vol. 89, no. 9, pp. 1541-1557, 2010.

[17] A. Pankov, "Standing waves for discrete nonlinear Schrödinger equations: sign-changing nonlinearities," Applicable Analysis, vol. 92, no. 2, pp. 308-317, 2013.

[18] A. Pankov and G. Zhang, "Standing wave solutions for discrete nonlinear Schrödinger equations with unbounded potentials and saturable nonlinearity," Journal of Mathematical Sciences, vol. 177, no. 1, pp. 71-82, 2011.

[19] M. Willem, Minimax Theorems, Progress in Nonlinear Differential Equations and their Applications, 24, Birkhäuser, Boston, Mass, USA, 1996.

[20] G. Zhang, "Breather solutions of the discrete nonlinear Schrödinger equations with unbounded potentials," Journal of Mathematical Physics, vol. 50, pp. 1-12, 2009.

[21] P. H. Rabinowitz, Minimax Methods in Critical Point Theory with Applications to Differential Equations, vol. 65 of CBMS Regional Conference Series in Mathematics, American Mathematical Society, Washington, DC, USA, 1986. 


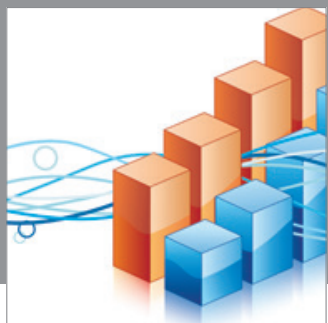

Advances in

Operations Research

mansans

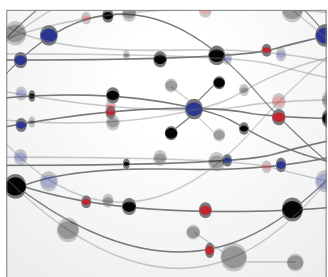

The Scientific World Journal
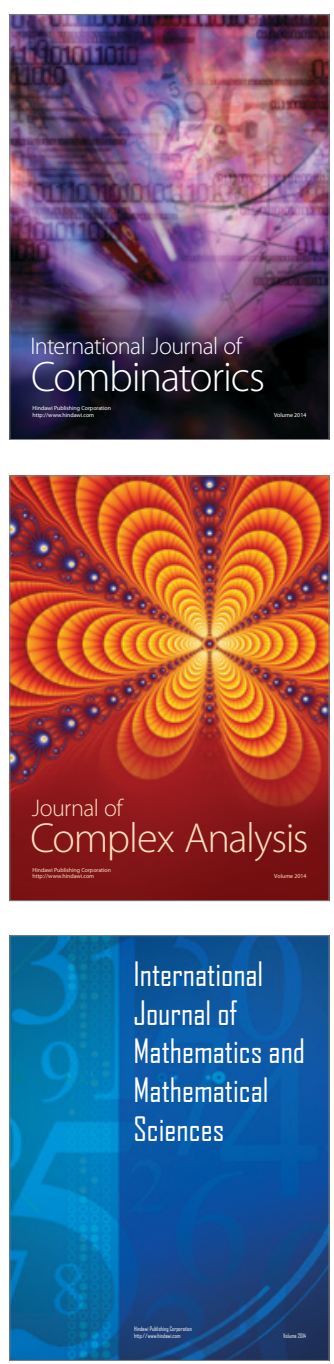
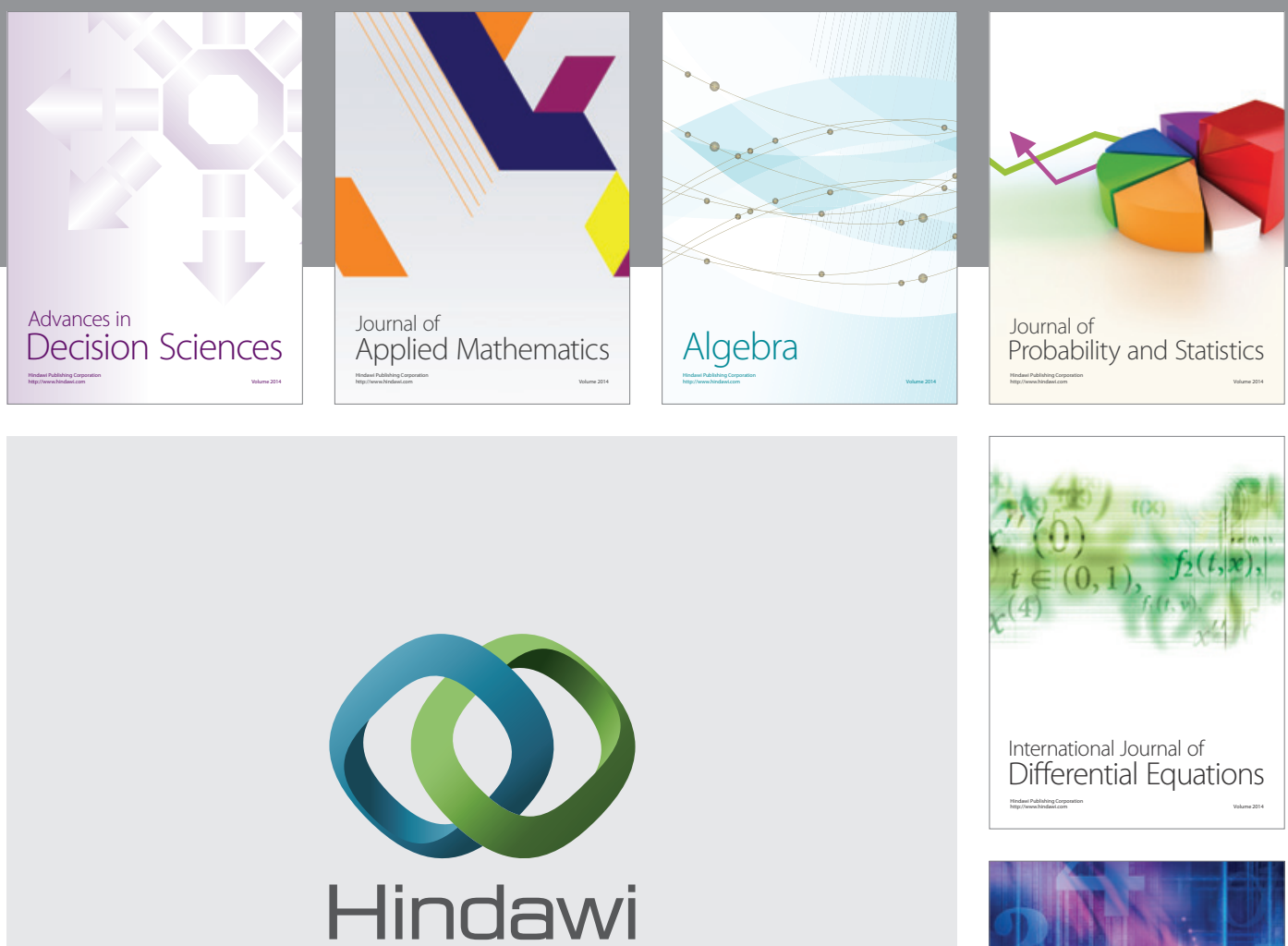

Submit your manuscripts at http://www.hindawi.com
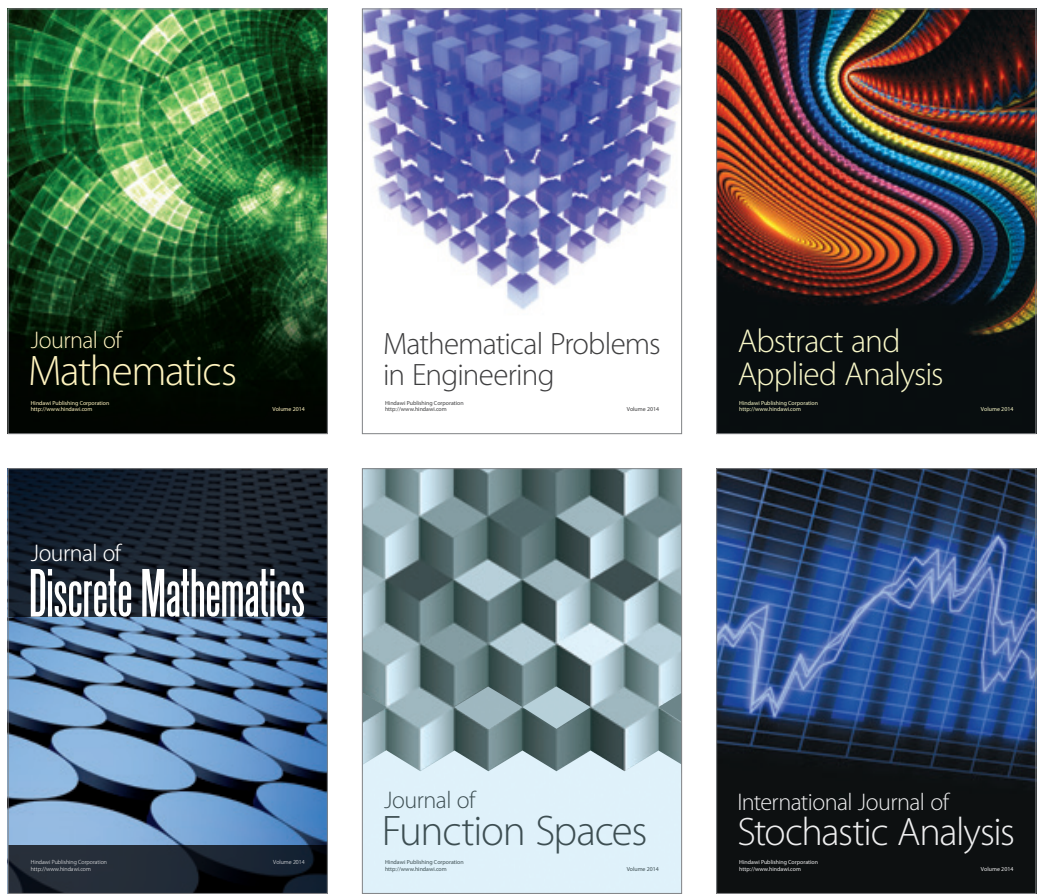

Journal of

Function Spaces

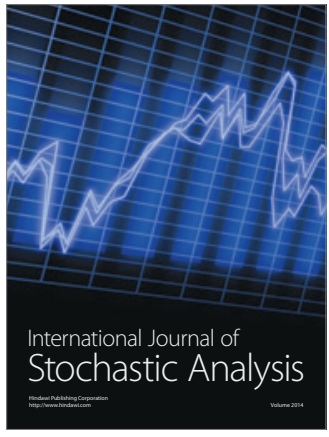

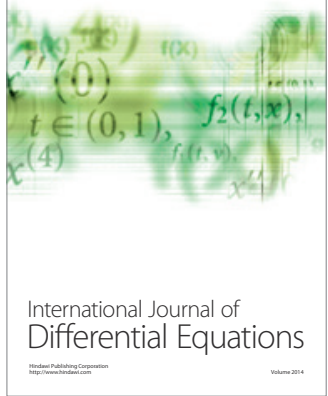
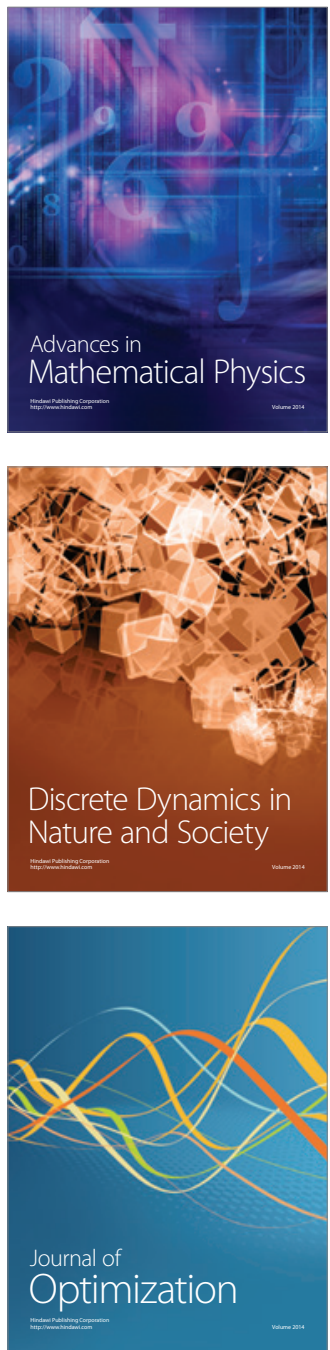